\title{
RESEARCH OF A HIGHLY DEFECTIVE FROG CORE OF GRADE 1/9
}

Pavels GAVRILOVS, Riga, Latvia, Riga Technical University, Institute of Railway Transport, 1 Paula Valdena Street, LV-1048, Latvia, pavels.gavrilovs@rtu.lv Viktors IVANOVS, Riga, Latvia, Riga Technical University, Institute of Railway Transport, 12 Āzenes Street, LV-1004, Latvia, viktori4301187@inbox.lv (corresponding author)

For the first time at the Riga Technical University a study was carried out of a highly defective frog core of grade 1/9. In the course of the research an analysis of crossing piece defects on the Latvian railway was carried out in eight railway sections during years of 2015, 2016, 2017. The defect of the frog core of grade 1/9 (the 60 E1 DO 0712 frog type) was considered according to the basic classification of the defects, and the analysis and research of the cause of its fracture were conducted from the bolt hole to the web and the base of the frog core.

The research process consisted of four stages:

- Stage 1: determination of metal hardness according to the Brinell scale with a modern device "Tinius O Olsen" Firmware Version 1.07, FH - 31 Series. The obtained results should be compared with the data of the manufacturer's factory - Dowlais Steel.

- Stage 2: determination of the chemical composition of the rail steel of the frog core 1/9 (in the rail top, rail web, and rail base) using the ARC-MET 8000 Mobile Lab Optical Emission Spectometer Analyser. The obtained data should be compared with the manufacturer's data.

- Stage 3: determination of the rail steel structure. Drawing of main conclusions about the quality of the rail steel of the frog core of grade $1 / 9$.

- Stage 4: drawing of main conclusions and summary of the cause and development of the fracture from the bolt hole of the frog core of grade $1 / 9$.

Keywords: analysis, base, chemical composition, core, defect, elements, frog, hardness, highly defective rail, mark, metal, top, web.

\section{INTRODUCTION}

On the Latvian railway point switches by the Austrian manufacturer "Dowlais Steel" are widely used in bolted rail type frogs. The frog core consists of two sections of rails attached to each other and connected by means of two wings and a set of liners with bolts (Figure 1). The frog of grade 1/9 is often placed on the reception and station routes of the Latvian railway. It allows trains to move along a straight path at speed up to $90 \mathrm{~km} / \mathrm{h}$, and from the main track to a diverging path at speed up to $25 \mathrm{~km} / \mathrm{h}$.

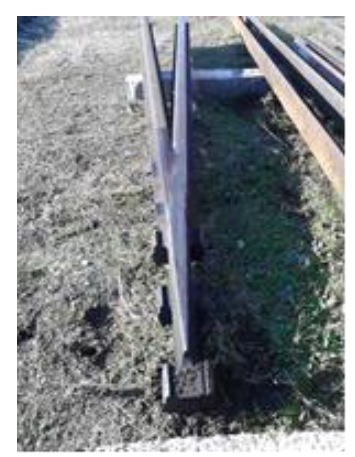

Figure 1. Frog core 1/9

On the Russian railways, frogs with cast cores are used in P65 type point switches of grade 1/9. They allow trains to move along a straight path at speed up to $100 \mathrm{~km} / \mathrm{h}$, and on diverging path at speed not exceeding $40 \mathrm{~km} / \mathrm{h}$. (GOST R 51685-2013 Rails 2014). 
The bolted frogs with a cast one-sided core of the general casting with the wearing part of the wings are commonly used among frogs with cast core in the Russian Federation (Figure 2). In such frogs, the core and wearing parts of the wings have single monolithic structure cast from high-manganese steel. The core is attached tothe wings, made of typical rails. The tread surface of cast parts of wings (Figure 2) is raised by $5 \mathrm{~mm}$ above the tread surface of the track rails, like the wings of solid frogs. These frogs, in comparison with the bolted-rail, have a longer service life and significantly fewer parts. Such frogs are widespread in the USA. At the same time, the connection of the frog members is less stable in comparison with the solid ones. In addition, the design is quite complicated; both curvature and profiled cutout is required during manufacturing wing rails.

In 2015, 41 highly defective pieces had been registered on the Latvian railway. However, the number of highly defective pieces decreased from 41 to 29 cases between 2015 and 2016 and then increased again to 39 cases in 2017 , as shown in the diagram (Figure 3).
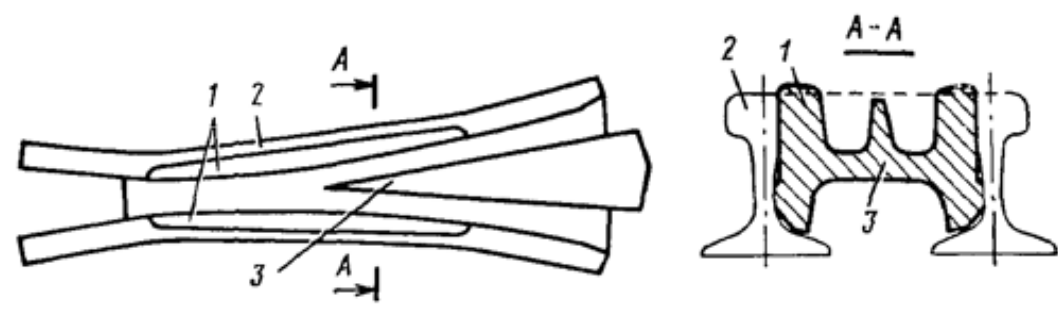

Figure 2. Bolted frog with cast core of general casting type with wearing part of wings: 1 - wearing parts of wings; 2 - wings; 3 - core.

The point switch frog is a special grooved structure designed to safely skip the crests of the wheels of the rolling stock at the intersection of internal rail joints where two paths converge on the point switch.

The frog consists of two main members:

- The core is a piece installed where internal rails converge.

- The wing is a piece which allows the wheel pair to roll up onto the core from the connecting rails.

The fracture of one of the listed members of the frog can cause the wheel pair to descend, as well as collapse of the rolling stock. Based on the statistical data, it was decided to study the highly defective frog core 1/9 of 60 E1 DO 07 12 type with code C.53.2. (Code C.53.2. is classified as C - in the core, 53 - crack in the web from bolted or other holes in the rails, 2 - is located outside the joint) (Baiko, 1997). It turned out that this problem is also relevant in other countries. In particular in Russian railways (RR) highly defective rail elements with code C.53.2 are rather common" (Rozhdestvenski, 1993).

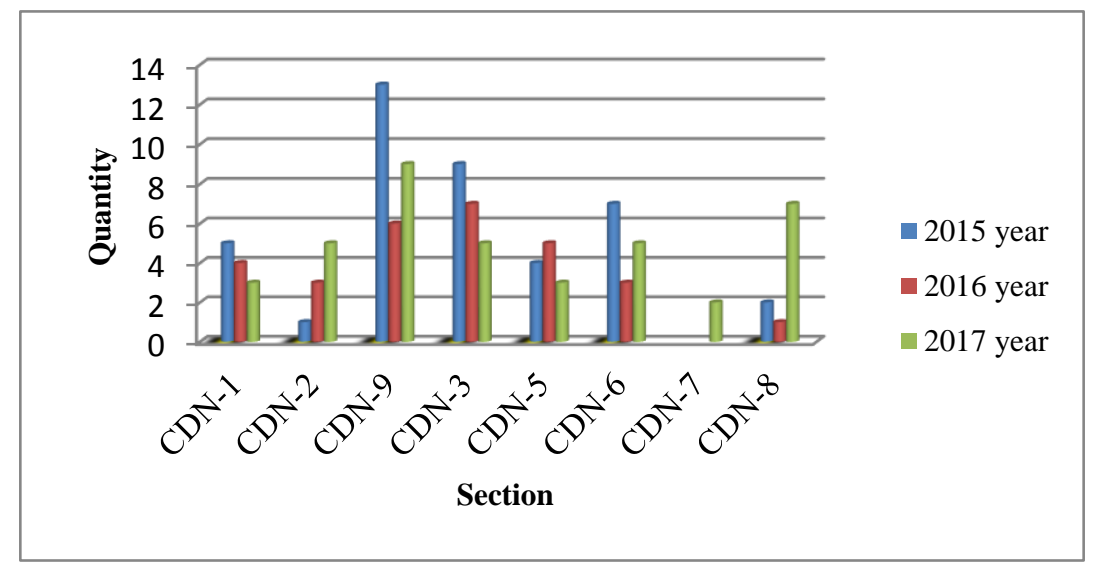

Figure 3. The diagram of highly defective rail members from 2015 to 2017

The Latvian railway is divided into eight sections of the route:

- CDN-1 Sorting Department, CDN-2 Ciekurkalna Department, CDN-9 Riga Department, CDN-3 Daugavpils Department,

- CDN-5 Rezekne Department, CDN-6 Ventspils Department, CDN-7 Lepaja Department, CDN-8 Jelgava Department.

It follows from the diagram (Figure 3) that in 2015 the largest number of rail defects was detected in the CDN-9 Riga Department (13), and rail defects were not found in the CDN-7 Lepaja Department. In 2016, rail defects (7) were detected in the CDN-3 Daugavpils Department. Rail defects were not found in the CDN-7 Liepaja Department during 2016. In 2017, the number of rail defects increased up to 39 cases; in particular, 9 rail defects were detected in the CDN9 Riga Department by workers of the flaw detection service. Two rail defects were detected in the CDN-7 Lepaja Department during 2017. 
The analysis of the statistics of the defects of point switches conducted on the railway network of JSC RR [ 13 . Rozhdestvenskii, 2008, pages 201-211 ] showed that the greatest number of defects were detected in the frog cores of point switches. Distribution of defects by the members of the point switches is shown in Figure 4.

- Defects of wing $(22 \%)$,

- Defects of switch point rails (15\%),

- Defects of core $(51 \%)$,

- Defects of point rail (11\%),

- Defects of track guard rail $(1 \%)$.

In accordance with NTD/CP-1-93 (Rozhdestvenskii, 1993), the defects in point switches to be identified are classified in the place of their formation: in the point rails (DR); in contact tongues (DO); in the connecting ways (of the same nature as in regular rails ); in the cores of solid frogs (DS, DU); in solid cores of frogs with welded rail ends; in the wings and cores of point switches with a continuous tread surface (DUN, DSN) (Strautmanis, 2016).

When analysing the problem of higly defective rail members on the railways, the question arises: what causes these defects? The first time in the laboratory of the Riga Technical University, it was decided to conduct a study of one of the members of the railway (a highly defective frog core 1/9 with code C.53.2) (Saikat, 2016) in order to analyse the condition of metal, to test its hardness and chemical composition, to determine its structure and compare the obtained data with the data from manufacturer's factory.

The defect code C.53. (Figure 4) is classified in the wing as cracks in the web from bolted or other holes in the rails. The defect code C.53. is subdivided into the C.53.1. and C.53.2. defect subcode, meaning that C.53.1 stands for a defect of the wing in the joint and C.53.2 stands for a defect of the wing located outside the joint. Stress concentration at the edges of bolt holes, absence or poor performance of chamfers, irregularities on hole surface due to poor drilling and corrosion accelerate development of cracks (Rozhdestvenski, 1993). Unsatisfactory condition of joints (weakening of bolts, crumpling and sagging of rail ends, subsidence in joints andpresence of large gaps) may be the main reason for the appearance and development of this type of defect (Pramanick, 2017).

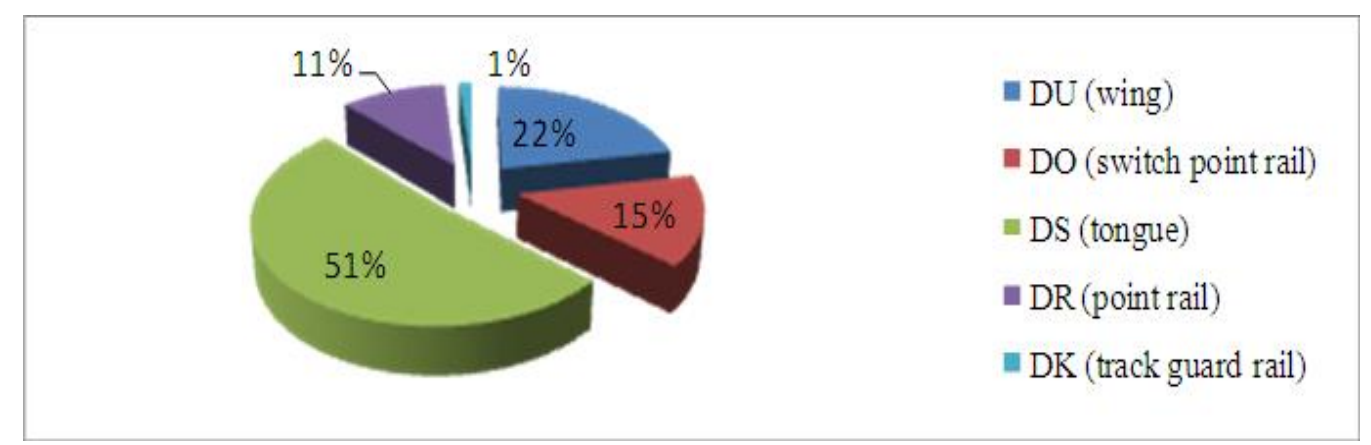

Figure 4. Distribution of defects in point switches in the places of their formation

The object of the research was a cut-out of highly defective frog core of grade 1/9 with the defect code C.53.2., the 60 E1 DO 0712 frog type, the UIC 60 rail type, manufactured in Dowlais Steel Works in April 2012. This specimen of the core of the fourth bolted hole (Figure 4) was cut from the frog throat out of the receiving and departure arrow No. 4 of the Vecumnieki line station of the Latvian Railway.

At Stage 1 of the research in the laboratory of the Riga Technical University, the rail steel hardness was tested according to the Brinell scale (HB) with device "Tinius O Olsen" Firmware Version 1.07, FH - 31 Series. The results of the research are shown in Figure 5. This made it possible to determine the hardness of the metal over the cross section of the core of the crosspiece and to give an accurate estimate of the quality of the production metal.

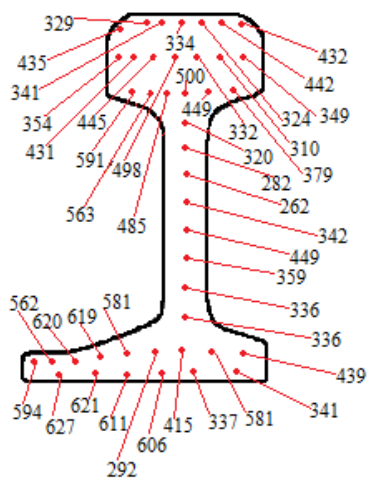

Figure.5. Determination of hardness of the frog core of grade 1/9 with 60 E1 DO 0712 frog type in HB: in core top:435, 329, 341, 334, 324, 442, 432, 354, 431, 445, 498, 332, 310, 349, 591, 563, 485, 500, 449, 379.

in core web: $320,282,262,342,449,359,336,336$.

in core base: 594, 562, 620, 619, 581, 292, 415, 581, 439, 627, 621, 611, 606, 337, 341 . 
On the basis of the data obtained, a table was compiled and comparisons were made with the manufacturer's passport data. The results of the comparisons are summarized in Table 1.

Table 1. Comparison of the hardness of 60 E1 DO core (the R350HT steel grade) with the manufacturer's data

\begin{tabular}{|l|c|c|}
\hline \multirow{2}{*}{\multicolumn{1}{c|}{ Hardness measurement area }} & \multicolumn{2}{c|}{ Rail hardness grade R350HT } \\
\cline { 2 - 3 } & Manufacturer's hardness (HB) & Medium hardness (the 60 E1 DO core) (HB) \\
\hline In HB top & 369 & 416 \\
\hline In web, not exceeding & 388 & 335 \\
\hline In base, not exceeding & 388 & 523 \\
\hline
\end{tabular}

The medium hardness in the core top is $416 \mathrm{HB}$, which is $47 \mathrm{HB}$ exceeding the permissible value of the manufacturer (Dijs, 2016). During the research the medium hardness in the core web was $335 \mathrm{HB}$, which was $53 \mathrm{HB}$ less. Based on the results obtained, the medium hardness in the base was $523 \mathrm{HB}$, which was $135 \mathrm{HB}$ more than in the manufacturer's rated values.

At Stage 2 of the research, the chemical composition of the metal was analysed at three points of the core (in top, web, and base). The specimen of a highly defective core was cut out with a circular saw from the frog core to be examined This specimen was polished to a mirror surface using a power sander (Figure 6).

To determine the chemical composition of the metal, an ARC-MET 8000 Mobile Lab optical emission spectometer analyser was used by means of local burning of the specimen surface followed by determination of chemical composition and obtaining of data on the printing device (Freimane, 2016). Measurements were made at three different points, three times, in the top, the web and the base of the wing. The average results of the analysis of the chemical composition of the specimens are given in Table 2. Thus, when researching the quality of metal, determining the chemical composition of the rail steel of the core of the crosspiece, is an integral part of the research process, since the main conclusions are based on the obtained chemical composition data in various countries of the world in particular and in the USA.
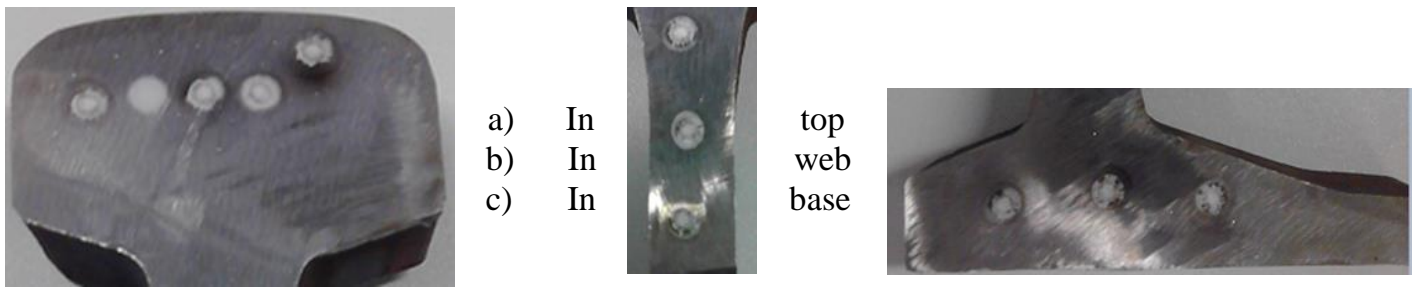

Figure.6 Determination the chemical composition in the three elements of the core

Table 2. The average and permissible values of the chemical composition (in percent ratio) of the examined frog core (top, web, and base) of grade 1/9 with $60 \mathrm{E} 1 \mathrm{DO} 0712$ frog type.

\begin{tabular}{|c|c|c|c|c|c|c|c|c|c|}
\hline Value & $\mathrm{Fe}$ & $\mathbf{C}$ & $\mathbf{S i}$ & Mn & $\mathbf{P}$ & $\mathbf{S}$ & $\mathrm{Cr}$ & Mo & $\mathbf{N i}$ \\
\hline $\begin{array}{c}\text { Max / } \\
\text { Min }\end{array}$ & - & $\pm 0,02$ & $\pm 0,02$ & $\pm 0,05$ & $+0,005$ & $+0,005$ & $\pm 0,02$ & - & - \\
\hline TOP & 97,3 & 0,976 & 0,399 & 1,13 & - & - & 0,0485 & 0,0100 & 0,0290 \\
\hline WEB & 97,1 & 0,965 & 0,402 & 1,08 & 0,125 & 0,0531 & 0,0520 & 0,0030 & 0,0233 \\
\hline BASE & 97,0 & 1,00 & 0,417 & 1,12 & 0,125 & 0,0587 & 0,0520 & 0,0030 & 0,0256 \\
\hline AVERAGe & 97,2 & 0,980 & 0,406 & 1,11 & 0,125 & 0,0559 & 0,0508 & 0,0050 & 0,0259 \\
\hline Value & Al & Co & $\mathrm{Cu}$ & $\mathrm{Nb}$ & $\mathbf{T i}$ & $\mathbf{V}$ & $\mathbf{W}$ & $\mathbf{P b}$ & $\mathbf{Z r}$ \\
\hline $\begin{array}{c}\text { Max / } \\
\text { Min }\end{array}$ & $+0,001$ & - & - & - & - & $+0,02$ & - & - & - \\
\hline TOP & 0,0068 & 0,0058 & 0,0253 & 0,0100 & 0,0050 & 0,0050 & 0,0400 & 0,0100 & - \\
\hline WEB & 0,0047 & 0,0050 & 0,0266 & 0,0030 & 0,0021 & 0,0043 & 0,0250 & 0,0100 & 0,0030 \\
\hline BASE & 0,0062 & 0,0050 & 0,0268 & 0,0030 & 0,0020 & 0,0051 & 0,0250 & 0,0004 & 0,0030 \\
\hline AVERAGE & 0,0059 & 0,0053 & 0,0262 & 0,0050 & 0,0030 & 0,0048 & 0,0300 & 0,0068 & 0,0030 \\
\hline
\end{tabular}

Table 3 shows the passport data of the chemical composition provided by Dowlais Steel factory.

Within the framework of the chemical analysis, the average statistical data of the tests carried out on three points of the examined core (tops, webs, and bases) were processed. The obtained data should be compared with the rated values of the manufacturer's (see Table 3 ).

In the course of determining the chemical composition of the core steel in the laboratory of the Riga Technical University at the Railway Department, the following differences were found (Korostelev, 1988). The following chemical elements were not indicated in the manufacturer's passport data: (Mo) molybdenum increases the red-hardness, elasticity, tensile strength, rust prevention and oxidation resistance at high temperatures, (Co) cobalt increases heat resistance, magnetic properties, increases shock resistance (Huang, 2014), (Nb) niobium improves acid resistance and contributes to 
reducing corrosion in welded structures, (Ti) titanium increases strength and density of steel, promotes grain refinement, acts as good deoxidizing agent, improves workability and corrosion resistance, (V) vanadium increases hardness and strength, refines the grain. Increases steel density, as it is a good deoxidizing agent, (W) wolframium forms carbides, very hard chemical compounds in the steel, which sharply increase hardness and red hardness. Wolframium prevents growth of the grains during heating, helps to reducing fragilidy during tempering, $(\mathrm{Pb})$ plumbum is a random impurity in the steel and has a significant effect on its mechanical and physical properties and also on corrosion resistance, (Zr) zirconium has a particular effect on the value and the growth of grain in steel, grinds the grain and allows to producte steel with a predefined granularity (Hua, 2017).

Table 3. Table of average values of the chemical composition provided by Dowlais Steel (the 60 E1 DO frog type) factory

\begin{tabular}{|c|c|c|c|c|c|c|c|c|c|}
\hline Value & C & $\mathbf{S i}$ & Mn & $\mathbf{P}$ & $\mathbf{S}$ & Al & H (ppm) & $\mathrm{Cr}$ & $\mathbf{N i}$ \\
\hline $\begin{array}{c}\text { Max / } \\
\text { Min } \\
\end{array}$ & $\pm 0,02$ & $\pm 0,02$ & $\pm 0,05$ & $+0,005$ & $+0,005$ & $+0,001$ & - & $\pm 0,02$ & - \\
\hline AVERAGE & 0,780 & 0,390 & 1,170 & 0,014 & 0,012 & 0,001 & 0,700 & 0,060 & 0,030 \\
\hline Value & $\mathrm{Cu}+10 \mathrm{Sn}$ & & & & & & & & \\
\hline $\begin{array}{c}\text { Max / } \\
\text { Min }\end{array}$ & - & & & & & & & & \\
\hline AVERAGE & 0,040 & & & & & & & & \\
\hline
\end{tabular}

Comparing the basic, permissible values of the chemical elements of the composition of rail steel (Table 2, Table 3 ), the following conclusion can be drawn: the carbon (C) content is increased by $0.18 \%$ if compared to manufacturer's data. The chemical element silicon $(\mathrm{Si})$ has the permissible percentage ratio and equals $0.406 \%$. Test results show that manganese $(\mathrm{Mn})$ content is normal (1.11\%). Ratio of harmful impurities such as phosphorus $(\mathrm{P})$ is increased 6.5 times, the excess being $0.106 \%$, as well as the content of sulphur (S) is increased 3 times and the excess being $0.0389 \%$. The content of aluminium (Al) was increased by $0.0039 \%$. The chromium $(\mathrm{Cr})$ content has the permissible percentage ratio and equals $0.0508 \%$ (Kapito, 2013). The percentage value of a chemical element sucha as nitrogen (N) was not revealed during the tests.

The performed tests allow determining the hardness of rail steel, the chemical composition of steel, to establish mass fraction of metal elements, and to answer the questions, for example, in case of rail fracture or cracking.

At Stage 3 of the research, the structure of rail steel (Marc, 2014) was determined with a modern electronic microscope Carl Zeiss Axiovert 40 MAT, feeding output data into the computer. The members (web and base) of the frog core were selected as specimens where open cracks were found. Metal structure was determined under the microscope at (x200) magnification (Molyneux, 2017).

Studies in Figure 7 allow drawing the main conclusions about the quality of the rail steel of the frog core 1/9. In the obtained data small inclusions in the form of black spots were found in the metal structure. This fact also confirms certain chemical composition. Comparing the metal structure at the x200 magnification (neck) and x200 magnification (sole), various inclusions were found and, it is well known that inclusions can be a "nucleus" of the formation of cracks. The metal structure considered is an unconfirmed confirmation of the investigation of the chemical composition of the quality of the metal of the core of the $1 / 9$ crosspiece.

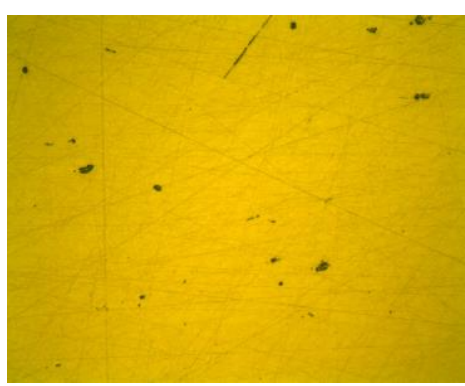

a) The web

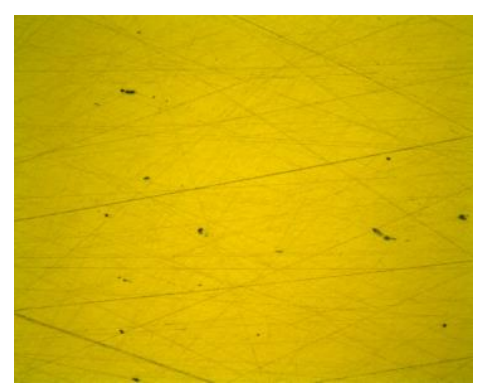

b) The base

Figure 7. Determination of the metal structure under a microscope in web and base at X200 magnification

\section{CONCLUSIONS}

1. In this paper, the main goal was achieved, namely hardness, chemical composition and metal structure of a highly defective core were measured and tested and the obtained data were compared with the manufacturer's standards.

2. Core steel hardness was tested according to the Brinell scale (HB) with the modern device "Tinius O Olsen" Fikrmware Version 1.07, FH - 31 Series. Metal hardness in the core top is $47 \mathrm{HB}$ higher than the permissible value of the factory standard.

3. The average hardness of the metal in the core web was $335 \mathrm{HB}$, which was $53 \mathrm{HB}$ less in the place of development of crack and the fracture from the bolt hole. 
4. Hardness in the base was $135 \mathrm{HB}$ higher than the manufacturer's standard.

5. The uneven distribution of the metal hardness from the top to the base, improper hardening of the metal, drilled hole and corrosion of the bolt hole were as one of the possible causes of weakening of the metal of the core web and the development of a crack due to a partial fracture of the frog core.

6. The chemical composition of the core steel in the top, web and base was determined. The obtained data were compared with the standard data. Results show deviations from the standards.

7. According to the studied data on the chemical composition of the frog core, the following conclusions can be drawn: the carbon (C) content is increased by $0.18 \%$. The chemical element silicon ( $\mathrm{Si}$ ) is in the permissible percentage ratio and equals $0.406 \%$. The manganese (Mn) content is normal and equals $1.11 \%$. The percentage content of phosphorus $(\mathrm{P})$ is increased 6.5 times and the excess equals $0.106 \%$, as well as the percentage content of sulphur $(\mathrm{S})$ is increased 3 times and the excess equals $0.0389 \%$. The aluminium $(\mathrm{Al})$ content is increased by $0.0039 \%$. Chromium $(\mathrm{Cr})$ has the permissible percentage ratio and equals $0.0508 \%$

8. Contents of such harmful chemical elements as (P) phosphorus and sulphur $(\mathrm{S})$ have non-accepted values and significantly affect the quality of the rail steel.

9. Wgeb analysing the metal of the frog core of grade $1 / 9$, a small number of inclusions were found.

10. Conducted research allows drawing a conclusion, that the possible reason of a partial fracture of a highly defective frog core of grade 1/9 had a number of factors, namely non-conformant metal hardness, prevailing harmful chemical elements such as sulphur $(\mathrm{S})$ and phosphorus $(\mathrm{P})$ in the metal, as well as the presence of small inclusions.

\section{REFERENCES}

1. Baiko, S. 1997. Latvian railway SJSC Service of railway equipment. Confirmed Latvian railway SJSC First deputy of CEO Norms and specifications for defective rails NTD-S-97. Riga, pp. 51-76.

2. Dijs, S., Tipainis, A. 2016. Assessment of railway turnout element restoration using mma and fcaw welding. Engineering for rural development. Latvia University of Agriculture Faculty of Engineering. 15th International Scientific Conference Engineering for Rural Development Proceedings, Vol. 15, pp. 606-611.

3. Freimane, J., Mezitis, M., Mihailovs, F. 2016. Maneuver Movements' Safety Increase Using Maneuver Locomotive Identification and Distance Control. Riga Technical University Riga; Latvia; pp. 375-379. https://doi.org/10.1016/j.procs.2017.01.148

4. GOST R 51685-2013 Rails. General technical specifications (as amended N1). National standard of Russian Federation Rails general technical specifications. 2014.

5. Huang, J. Xia, L., Youshou Z., Sinian, L.. 2014. Investigation on brittle fracture mechanism of a grade E cast steel knuckle. Journal Case Studies in Engineering Failure Analysis, Vol. 2, pp. 15-24. https://doi.org/10.2351/1.4983232

6. Hua, H., Ting Z., Mingxing Ma, and Wenjin Liu. 2017. Microstructure and wear resistance of laser cladding particulate reinforced Fe-based composite coating on railway steel. Journal of Laser Applications, Vol. 29, No. 2, pp. 1-5.

7. Korostelev, P.P. 1988. Reagents for technical analysis. 104 pages. https://doi.org/10.2351/1.4983232

8. Kapito, A., Stumpf, W., Papo, M.J. 2013. The role of alloying elements in bainitic rail steels. The Journal of the Southern African Institute of Mining and Metallurgy, Vol. 113, pp. 67-72 pages https://doi.org/10.2351/1.4983232

9. Marc, A., Cameron, D., Arthur, E., 2014. Purslow, E.W.I., 1250. Adams Drive, Columbus, OH 43221. 614.688.5150; Stuart, Federal Railroad Administration, 1200 New Jersey Avenue S.E., Washington, D.C. 20590 202.493.6384; Yim Har Tang, Volpe National Transportation Systems Center, Kendall Square, Cambridge, MA 02142-1092 617.494.2717. Weld Repair of Manganese Frogs for Enhanced Safety in Shared Service. Proceedings of the Arema 2014 Annual Conference \& Exposition Chicago, pp. 1-20.

10. Molyneux-Berry, P., Claire, D., Bevan., A. 2017. The Influence of Wheel/Rail Contact Conditions on the Microstructure and Hardness of Railway Wheels. The Scientific World Journal, Vol. 214, pp. 1-16.

11. Pramanick, A.K., Das, G., Das, S.K., Ghosh, M. 2017. Failure investigation of super heater tubes of coal fired power plant. Journal Case Studies in Engineering Failure Analysis, Vol. 9, pp. 17-26.

12. Rozhdestvenskii, S. A. 1993. NTD/CP 1-2-3-93 Classification of rail defects. Catalog of defects of rails. Characteristics of defective and highly defective rails. - M.: Transport, 1993. 64 p.

13. Rozhdestvenskii, S. A. 2008. UDC 620.19 non-destructive testing of elements of point switches. News of PSTU, No. 1, pp. $201-211$.

14. Rozhdestvenskii, S. A. 1993. Addition to the NTD/CP 1-2-3-93. Classification of defects and damages of elements and damages of point switches. Catalog of defects and damages of elements of point switches. Characteristics of defective and highly defective elements of point switches. - M.: Transport, $65 \mathrm{p}$.

15. Strautmanis, G., Mezitis, M., Strautmane, V. 2016. Model of a vertical rotor with a ball-type automatic balancer. 22nd International Conference on Vibroengineering; Moscow; Russian Federation; 4 October 2016 - 7 October, pp. 57-62.

16. Kumar, De S., Srikanth, S., Bhakat, A. K., Saxena Atul, Jha B. K. 2016. Copper Bearing Steels from SAIL and Its Application. International Journal of Metallurgical Engineering, Vol. 5(1), pp. 1-8. 